\title{
Questes
}

vestes Revue pluridisciplinaire d'études médiévales

\section{7 | 2018}

\section{Sexualités et interdits}

\section{Introduction}

\section{Élodie Guilhem et Catherine Kikuchi}

\section{(2) OpenEdition \\ Journals}

Édition électronique

URL : http://journals.openedition.org/questes/4459

DOI : 10.4000/questes.4459

ISSN : 2109-9472

\section{Éditeur}

Les Amis de Questes

\section{Édition imprimée}

Date de publication : 31 janvier 2018

Pagination : 9-32

ISSN : 2102-7188

\section{Référence électronique}

Élodie Guilhem et Catherine Kikuchi, « Introduction », Questes [En ligne], 37 | 2018, mis en ligne le 01 février 2018, consulté le 22 avril 2019. URL : http://journals.openedition.org/questes/4459; DOI :

10.4000 /questes.4459 


\section{Introduction}

\section{Élodie GUILHEM et Catherine KIKUCHI}

EPHE et École française de Rome

Commençons par une représentation de la sexualité au Paradis donnée par un théologien du $\mathrm{IX}^{\mathrm{e}}$ siècle, Jean Scot Érigène. Celui-ci affirmait contre l'avis d'autres théologiens que la sexualité existait entre Adam et Ève. Selon lui, Adam était capable de mouvoir tous les membres de son corps par sa seule volonté. Il pouvait alors pénétrer «le flanc» d'Ève, avec calme, et permettre à sa semence, toujours sous l'effet de sa volonté, de féconder le ventre de celle qui lui avait été donnée pour épouse : «Dans la tranquillité du corps et de l'âme, sans la corruption de la virginité, le mari avait, ou plutôt aurait eu la possibilité de féconder le flanc de son épouse ${ }^{1} \gg$.

C'est bien une sexualité sans désir, sans plaisir, et donc sans péché, que l'on nous propose. Mais de la Chute a découlé une sexualité passionnée, mue par la recherche de la satisfaction des sens et accompagnée de gestes frénétiques bien loin de la sérénité du jardin d'Eden. Ainsi, dans une certaine mesure, on pourrait considérer tous les interdits touchant la sexualité chrétienne médiévale comme une recherche de cette copulatio sans péché qui était celle du couple originel. Ce que l'on sait de la sexualité est cependant tellement dépendant des textes normatifs, punitif, et de la littérature courtoise ou grivoise, que nous avons souhaité étudier la sexualité sous l'angle des interdits.

\footnotetext{
${ }^{1}$ Patrologie latine, 122, 806, cité dans Georges Duby, La Chevalier, la femme et le prêtre. Le mariage dans la France féodale, Paris, Hachette, 1981, p. 56-57.
} 
Qu'est-ce que la sexualité ? Selon Michel Foucault repris par l'historien Jacques Rossiaud, une sexualité, du point de vue de la société, est une structure complexe faite de trois ensembles liés : des pratiques multiples plus ou moins codées, mais toujours perçues comme licites ou illicites ; une scientia sexualis qui énonce les normes et les déviances ; un ars erotica, produit de l'expérience, des réactions à la norme, à sa transgression comme de la propagande normative ${ }^{2}$. Les sexualités se comprennent ainsi surtout par rapport aux normes et donc aux interdits qui prétendent les diriger. Parler de la sexualité en lien avec ces derniers, c'est finalement faire l'hypothèse que la sexualité se définit socialement, à travers les normes et le rapport aux normes, à travers un système répressif puis une réappropriation, qui informent aussi la manière dont sont organisées et dont se pensent les sociétés. La sexualité de l'individu est contrainte et conditionnée aux normes de la société.

Nous avons choisi de prendre comme point de départ les normes religieuses. Les interdits posés par les textes religieux et leurs interprétations infusent la société médiévale. Nous nous intéresserons dans ce cadre aux trois religions du Livre, qui présentent des visages relativement différents du rapport à la sexualité et aux interdits qui la règlent.

\section{Les normes}

\section{Le judaïsme}

Les normes sexuelles de l'Ancien Testament sont mentionnées dans de nombreux passages et ne peuvent être réduites à la simple injonction du Décalogue contre l'adultère. De nombreux textes de la Torah présentent des distinctions fermes entre licite et illicite, entre le pur

2 Michel Foucault, Histoire de la sexualité, Paris, Gallimard, 1976, 3 t., et Jacques Rossiaud, Sexualités au Moyen Âge, Paris, Gisserot, 2012, p. 4-5. 
et l'impur. D'abord l'Écclésiaste : «Il y a un temps pour embrasser et un temps pour éviter d'embrasser ${ }^{3} »$. À partir de cette injonction se greffent une série d'empêchements, notamment dans le Lévitique ou les Livres des Prophètes, qui introduisent des interdits sexuels formels liés à l'impureté de certains temps, de certains actes ou de certaines personnes. Ainsi, le Lévitique règlemente de façon très stricte l'interdiction des rapports sexuels entre époux au moment des menstruations ou après l'accouchement.

Quand une femme est atteinte d'un écoulement, que du sang s'écoule de ses organes, elle est pour sept jours dans son indisposition, et quiconque la touche est impur jusqu'au soir [...] Si un homme va jusqu'à coucher avec elle, elle lui transmet son indisposition : il est impur pour sept jours ${ }^{4}$.

Quand un homme couche avec une femme qui a ses règles et qu'il découvre sa nudité, puisqu'il a mis à nu la source du sang qu'elle perd, et qu'elle-même a découvert cette source, ils seront tous les deux retranchés du sein du peuple ${ }^{5}$.

La même impureté touche l'accouchée «comme dans le cas de son indisposition menstruelle » et elle doit attendre puis faire accomplir par le prêtre le rite d'absolution par lequel «elle est purifiée de sa perte de $\operatorname{sang}^{6} \gg$.

On voit que la notion de pureté est ici primordiale. Il ne s'agit pas d'une simple injonction sanitaire. Cet interdit a une nature fondamentalement religieuse : les Hébreux, peuple de Dieu, doivent être purs pour ne pas encourir le châtiment divin. Coucher avec une

\footnotetext{
${ }^{3}$ Écclésiaste III:5, dans Ancien Testament, Traduction œcuménique de la Bible, Paris, Cerf/Les Bergers et les Mages, 1976.

${ }^{4}$ Lévitique XV:19-24, dans Ibid.

${ }^{5}$ Lévitique XX:18, dans Ibid.

${ }^{6}$ Lévitique XII:1-7, dans Ibid.
} 
menstrueuse rend impur; ce contact charnel est dangereux à cause de Dieu et il souille le peuple élu tout entier?

Cette impureté peut être comprise comme le résultat de l'acte charnel en soi. Il s'agit d'une position défendue par certains courants kabbalistiques médiévaux. Selon eux, Dieu étant asexuel et purement spirituel, il faut se détacher du corps et du sexe pour se rapprocher du divin. Ce courant ascétique est contrebalancé dans les études kabbalistiques par un autre, qui au contraire insiste sur l'importance de l'imagerie sexuelle pour comprendre le monde supranaturel. Que l'on pense par exemple au Cantique des Cantiques qui utilise la métaphore de l'amour humain et charnel pour parler de l'amour de Dieu : «Qu'il m'embrasse à pleine bouche ! Car tes caresses sont meilleures que du vin, meilleures que la senteur de tes parfums ${ }^{8} \gg$. Selon ce courant, l'homme est défini par sa différenciation sexuelle, qui reflète la division du monde divin. Le corps prend alors une grande importance pour la vie religieuse ${ }^{9}$.

\section{Le christianisme}

Le christianisme reprend très largement les interdits calendaires touchant les relations sexuelles dans le judaïsme, tout en apportant des conceptions différentes de l'acte charnel.

Les interdits sexuels chrétiens ont été largement étudiés par une bibliographie abondante, marquée notamment par les travaux de Georges

\footnotetext{
${ }^{7}$ Jean-Louis Flandrin, Un Temps pour embrasser. Aux origines de la morale sexuelle occidentale ( $\mathrm{VI}^{\mathrm{e}}-\mathrm{XI}$ siècle), Paris, Éditions du Seuil, 1983, p. 77-78.

${ }^{8}$ Cantique des cantiques, I:2-3, dans Ancien Testament, op. cit.

${ }^{9}$ Il s'agit d'une piste de recherche qui a été travaillée par deux études principalement : Moshe Idel, Kabbalah and Eros, New Haven/Londres, Yale University Press, 2005. Voir aussi David Biale, Eros and the Jews : from biblical Israel to contemporary America, Berkeley/Los Angeles/Londres, University of California Press, 1997. Voir également les explications claires et synthétiques d'Adin Steinsaltz, dans La Rose aux treize pétales. Introduction à la Cabbale et au judaïsme, Paris, Albin Michel, 2002, p. $178-180$.
} 
Duby et de Jean-Louis Flandrin ${ }^{10}$. Si les Évangiles sont peu loquaces à ce sujet, saint Paul puis les Pères de l'Église se sont montrés attentifs à réglementer une sexualité perçue dès le départ comme mauvaise. Pour Grégoire de Nysse, la différenciation sexuelle et la sexualité sont des conséquences du péché originel et représentent des caractéristiques bestiales de l'homme; le mariage et le mode de reproduction sexué sont donc des conséquences de la Chute ${ }^{11}$. De même, «saint Augustin [...] considérait le plaisir sexuel comme inévitablement vicié par la concupiscence, et voyait dans l'œuvre de procréation le bien nécessaire de l'acte conjugal ${ }^{12} »$. Pour les Pères de l'Église, tout acte qui n'aurait pas cette finalité est un péché d'adultère ou de fornication : selon saint Jérôme, «adultère est aussi l'amoureux trop ardent de sa femme » et «rien n'est plus honteux que d'aimer une épouse comme une maitresse ${ }^{13} »$. Ces positions sont cependant nuancées plus tardivement, notamment par Thomas d'Aquin, qui lie le plaisir à l'acte conjugal ayant pour fin la procréation, ce qui ne l'empêche pas de condamner l'acte sexuel qui aurait le plaisir pour seule fin ${ }^{14}$. Mais avant la scolastique, les écrits des Pères de l'Église antique et du haut Moyen Âge sont avant tout

\footnotetext{
${ }^{10}$ Avec deux ouvrages fondateurs : Georges Duby, Le Chevalier, la femme et le prêtre, op. cit. et deux ans après, Jean-Louis Flandrin, Un Temps pour embrasser, op. cit. Le premier couvre les $\mathrm{XI}^{\mathrm{e}}$ et $\mathrm{XII}^{\mathrm{e}}$ siècles, alors que Flandrin traite de la morale sexuelle présentée notamment dans les pénitentiels entre les $\mathrm{VI}^{\mathrm{e}}$ et $\mathrm{XI}^{\mathrm{e}}$ siècles.

${ }^{11}$ Grégoire de Nysse, La Création de l'homme, trad. Jean Laplace, Paris/Lyon, Cerf/Édition de l'Abeille, 2002 [1944]. Voir à ce sujet Fernard Floeri, « Le sens de la "division des sexes" chez Grégoire de Nysse », Revue des Sciences Religieuses, t. 27, fasc. 2, 1953, p. 105-111 et Édouard Jeaneau, «La division des sexes chez Grégoire de Nysse et chez Jean Scot Érigène », dans Études érigéniennes, 1987, p. 341-364.

12 Jean-Louis Flandrin, «Contraception, mariage et relations amoureuses dans l'Occident chrétien », Annales. Économies, Sociétés, Civilisations, vol. 24, n 6, 1969, p. 1370-1390, cit. p. 1379.

${ }^{13}$ Saint Jérôme, Adversus Jovinianum, I, 49 ; Patrologie latine, 23, col. 280-281.

14 Thomas d'Aquin, De Malo, 15, 2, analysé par John Thomas Noonan dans Contraception et mariage : évolution ou contradiction dans la pensée chrétienne, Paris, Cerf, 1969. Sur ces évolutions, voir en particulier Jean-Louis Flandrin, «Contraception, mariage et relations amoureuses dans l'Occident chrétien », art. cit. ainsi que Georges Duby, Le Chevalier, la femme et le prêtre, op. cit., p. 30-33.
} 
des écrits de moines, pétris de culture gréco-latine, et combinent une reprise des interdits hébraïques avec une influence forte de l'ascétisme et notamment de la philosophie stoïcienne ${ }^{15}$. La chasteté est le but à atteindre pour se rapprocher de Dieu ; si on ne peut atteindre cet objectif, l'acte sexuel dans le mariage doit bannir le plaisir, satisfaire les besoins des conjoints tout en visant au « croissez et multipliez » biblique.

À l'âge féodal, la règlementation sociale de la sexualité prend le dessus. On voit les clercs tout occupés à règlementer un mariage indissoluble qui peine à s'imposer en Europe. Il s'agit de lutter contre les formes de concubinages, notamment les mariages more danico. Dans le même temps, ils s'acharnent à traquer les formes d'inceste si courantes dans les classes dominantes ${ }^{16}$. La norme est fixée au concile de Latran IV en 1215 : on interdit le mariage entre parents au quatrième degré ${ }^{17}$. Les historiographes ou hagiographes s'attachent ainsi à raconter la lutte des clercs contre les mariages de trop proche parenté, mais ils rapportent aussi la vertu des épouses soumises comme il se doit à leur mari, souvent tourmentées par les adultères, les rejets et les mauvais traitements. Ainsi en Flandre, Godelive, dont l'hagiographie fut rédigée en $1084^{18}$, est poussée à la fuite par les mauvais traitements puis assassinée par son mari, ou encore Ide, comtesse de Boulogne, qui usa du mariage avec la

\footnotetext{
${ }^{15}$ Jean-Louis Flandrin, Un Temps pour embrasser, op. cit., chap. III « Origines et raisons des interdits », p. 72-127.

${ }^{16}$ Georges Duby, Le Chevalier, la femme et le prêtre, op. cit.

${ }^{17}$ Sur les normes du mariage et de la parenté en Occident, on se réfèrera notamment à Jack Goody, L'Évolution de la famille et du mariage en Europe, Paris, Armand Colin, 1985 ; Anita Guerreau-Jalabert, «Sur les structures de parenté dans l'Europe médiévale », Annales. Économie, Société, Civilisation, 1981, vol. 36, nº 6, p. 1028 1049 et Jean Gaudemet, Le Mariage en Occident : les mours et le droit, Paris, Cerf, 1987.

${ }^{18}$ Acta Sanctorum, Juillet II, 403, cité dans Georges Duby, Le Chevalier, la femme et le prêtre, op. cit., p. 142-147.
} 
plus grande chasteté, en vue de la procréation et dans la soumission à son $\operatorname{mari}^{19}$.

On observe cependant une inflexion à partir de 1200 avec une valorisation nouvelle du corps face aux hérésies, notamment contre les Parfaits cathares qui tendent à rejeter les aspects matériels, liés au démon, par opposition à l'esprit, lié à Dieu ${ }^{20}$. En réaction, on voit également se développer de plus en plus une piété insistant sur la corporalité du Christ dans son incarnation, notamment dans la nouvelle importance du sacrement de l'eucharistie ${ }^{21}$. Les clercs vont alors chercher à donner une sainteté au mariage et à l'état conjugal. Pour certains, il devient même licite de chercher le plaisir au sein du mariage, reprenant des réflexions de saint Augustin ou d'Abélard. Ainsi Gerson : «L'époux qui recherche le plaisir charnel sans autre fin ne pêche pas mortellement ». Mais il va plus loin encore : «La mariée doit penser à plaire à son mari et le mari à sa femme, selon commun profit pour le mariage ${ }^{22} »$. À la fin du Moyen Âge, la «conversation charnelle » au sein du mariage est moins empreinte de soupçon. Les autorités ecclésiastiques se tournent alors vers un autre interdit qui avait été peu mis en avant dans les premiers siècles du Moyen Âge : l'homosexualité, désormais réprimée avec bien plus d'ardeur.

\footnotetext{
${ }^{19}$ Acta Sanctorum, Avril I, 141-144, cité dans Georges Duby, Le Chevalier, la femme et le prêtre, op. cit., p. 147-150.

${ }^{20}$ Voir Jean-Louis Biget, Hérésie et inquisition dans le Midi de la France, Paris, Picard, 2007.

${ }^{21}$ André Vauchez, La Spiritualité du Moyen Âge occidental, VIII-XIII ${ }^{\mathrm{e}}$ siècle, Paris, Seuil, 1994, chap. 5 «L'homme médiéval à la recherche de Dieu », p. 169-189.

22 Jean Gerson, Euvres complètes, éd. Pharamond Glorieux, Paris, Desclée, 1962, t. VII, p. 855, cité dans Jacques Rossiaud, Amours vénales. La prostitution en Occident, $\mathrm{XII}^{\mathrm{e}}-\mathrm{XVI}^{\mathrm{e}}$ siècle, Paris, Aubier, 2010, p. 62 ; sur cette évolution de la position des théologiens, voir ibid., p. 55-69.
} 


\section{L'islam}

Le Coran présente une autre vision de la sexualité. L'union charnelle ne possède pas de caractère honteux, tant qu'elle reste dans le cadre du mariage. Au contraire, la sexualité prend une dimension religieuse: les deux sexes sont la réflexion de l'organisation de la Création, la sexualité permet ainsi d'atteindre le divin et de reconnaître la puissance de Dieu; l'acte sexuel est un devoir, mais un devoir joyeux, avec le plaisir qui l'accompagne et qui doit être partagé par les deux conjoints. Les interdits bibliques subsistent bien sûr: la fornication («Évitez la fornication : c'est une abomination $!^{23} »$ ), 1'homosexualité masculine («Souvenez-vous de Loth ! [...] Vous vous approchez des hommes de préférence aux femmes pour assouvir vos passions. Vous êtes un peuple pervers $\left.{ }^{24} »\right)$, l'adultère, l'inceste qui est défini assez largement («Vous sont interdites vos mères, vos filles, vos sœurs, etc. $\left.{ }^{25} »\right)$ et la prostitution ( « Ne forcez pas vos femmes esclaves à se prostituer pour vous procurer les biens de ce monde, alors qu'elles voudraient rester honnêtes $\left.{ }^{26} »\right)$. Mais «hormis les interdictions mentionnées, il vous est permis de satisfaire vos désirs, en utilisant vos biens d'une façon honnête et sans vous livrer à la débauche ${ }^{27} \gg$.

Même l'acte sexuel est codifié de façon hédoniste, en prescrivant des préliminaires, des «baisers et douces paroles », et en proscrivant de «se jeter sur sa femme comme le font les bêtes ${ }^{28}$ ». La jouissance de sa femme est un devoir pour le mari. L'ascétisme, au contraire, est

\footnotetext{
${ }^{23}$ Le Coran, éd. et trad. Denise Masson, Paris, Gallimard, 1980, XVII, 32. Sur le commentaire des préceptes de l'Islam concernant la sexualité, on se réfèrera à Abdelwahab Bouhdiba, La Sexualité en islam, Paris, PUF, 1975 ainsi qu'à GeorgesHenri Bousquet, L'Éthique sexuelle de l'Islam, Paris, Desclée de Brouwer, 1966.

${ }^{24}$ Le Coran, op. cit., VII, 80-81.

${ }^{25}$ Ibid., IV, 33.

${ }^{26}$ Ibid., XXIV, 33.

${ }^{27}$ Ibid., IV, 34.

${ }^{28}$ Ibid., II, 223.
} 
découragé, voire moqué, sauf dans certains courants notamment soufis ${ }^{29}$. Contrairement au christianisme, des méthodes de contrôle des naissances et l'avortement sont bien plus largement tolérés ${ }^{30}$.

Les lois de l'islam règlementent finalement le plaisir et l'acte sexuel pour éviter les débordements et préserver la vie dans la société et la complémentarité des sexes dans celles-ci. Par exemple, le travestissement est fondamentalement réprimé, puisqu'il brouille l'ordre social et l'ordre voulu par Dieu. Les interdits sexuels sont avant tout des interdits sociaux et politiques, distinguant entre les relations licites et illicites. Certains actes sont marqués du sceau du tabou, zin, et sont punis dans le Coran par un châtiment apocalyptique : le viol en représente le péché capital ${ }^{31}$.

Ces interdits sont ce qu'ils sont : prescriptifs, réappropriés ou non par les acteurs. Une des manières d'observer cette réappropriation, l'intégration ou la déformation de ces normes, serait à présent d'observer les représentations, littéraires ou visuelles, des interdits sexuels.

\section{Les représentations}

Les artistes tentent dans leurs représentations de figurer les interdits sexuels ; mais l'image est en partie censurée pour enseigner sans danger les interdits : elle a une portée moralisatrice et éducative ${ }^{32}$. À partir du XIII ${ }^{\mathrm{e}}$ siècle, l'étude de la nature, la connaissance des sciences antiques via

\footnotetext{
${ }^{29}$ Notamment les maîtres soufis Abû Hamid Ghazâli (1058-111) et Ibn al Faridh (1181-1235) : Jean Chevalier, Le Soufisme, Paris, PUF, 1984, p. 45-55.

${ }^{30}$ Abdelwahab Bouhdiba, La Sexualité en islam, op. cit., p. 154-155.

31 «Zina», dans Malek Chebel, Encyclopédie de l'amour en Islam, vol. 2, Paris, Payot \& Rivages, 2003, p. 423 ; Abdelwahab Bouhdiba, La Sexualité en islam, op. cit., p. 25 et Georges-Henri Bousquet, L'Éthique sexuelle de l'Islam, op. cit., p. 63.

32 Michel Pastoureau, «Introduction », dans Florence Colin-Goguel L'Image de l'amour charnel au Moyen Âge, Paris, Éditions du Seuil, 2008, p. 11.
} 
les intellectuels arabes et les commandes des laïcs entraînent la création de nouvelles représentations de la sexualité ${ }^{33}$.

\section{La sexualité et le sacré}

Sur de nombreuses œuvres d'art, même celles à destination religieuse, apparaissent des scènes obscènes, qui sont probablement inspirées de l'Antiquité. Ces représentations avaient une valeur prophylactique $^{34}$. Ainsi, la tenture de la reine Mathilde ${ }^{35}$, réalisée en 1073 pour la consécration de la cathédrale de Bayeux, devait marquer la légitimité au trône d'Angleterre de Guillaume le Conquérant. Dans les marges figure un bestiaire fantastique mais également des personnages nus. Ces images, au registre inférieur, coïncident avec le personnage de Harold, au registre médian ; elles pourraient donc symboliser la traîtrise du personnage. Sur la tapisserie de Bayeux figure une scène encore plus énigmatique et choquante qui s'intitule « où un clerc et Aelfgyva». Dans cette image, le clerc relève le menton de la femme. Il s'agit d'un acte outrageant, figurant un clerc en train de rompre ses vœux par le viol ou le rapt d'une jeune femme. Au registre inférieur se tient un homme nu dans une attitude ithyphallique, ce qui confirme cette hypothèse. Cette image évoque par ailleurs un épisode historique, les protagonistes étant nommés ${ }^{36}$. Cette scène particulière renverrait à un contexte plus général, en effet, au XI $\mathrm{XI}^{\mathrm{e}}$ siècle, les clercs sont préférés aux chevaliers comme

\footnotetext{
33 Ibid.

${ }^{34}$ Arnaud de la Croix, L'Érotisme au Moyen Âge. Le corps, le désir et l'amour, Paris, Tallandier, 1999, p. 14 et Denis Bruna, Saints et Diables au chapeau, Paris, Éditions du Seuil, 2007, p. 155-157. Certaines enseignes représentent des phallus ou des vulves, elles seraient en réalité des amulettes protectrices contre le mauvais œil, mais elles évoqueraient aussi des cultes liés à la fécondité issus de la tradition antique.

${ }^{35}$ Cette tenture est couramment appelée la tapisserie de Bayeux. Il s'agit en réalité d'une broderie.

${ }^{36}$ Michael Camille, L'Art de l'amour au Moyen Âge. Objets et sujets du désir, Cologne, Könemann, 2000, p. 16-18 et Florence Colin-Goguel, L'Image de l'amour charnel au Moyen Âge, op. cit., p. 94-95.
} 
amants car ils sont plus raffinés dans leurs manières ${ }^{37}$. La mise en place de nouveaux interdits par l'Église comme le mariage des prêtres ou encore l'institution du mariage comme sacrement sont postérieurs à cette broderie.

La portée symbolique de ces images change au XI ${ }^{\mathrm{e}}$ siècle ; elles deviennent le symbole de l'Enfer et de la mort, représentant les péchés capitaux, en particulier la Luxure. Ainsi sur le tympan de l'église SaintJacques de Compostelle, dans la scène de la tentation du Christ : une femme tient un crâne, elle rappelle donc la faute commise par Adam tout en rappelant par son apparence la figure de Méduse. Elle associe dès lors le péché originel et la mort. L'Espagne chrétienne montre d'ailleurs beaucoup d'exemples de cette iconographie ancienne réinterprétée. Les Bénédictins qui organisent le pèlerinage vers ce sanctuaire éditent au XII ${ }^{\mathrm{e}}$ siècle un Guide du pèlerin, dans lequel, on peut lire « Et il ne faut pas oublier de mentionner la femme qui se trouve à côté de la tentation du Christ, tenant entre ses mains la tête fétide de son lécheur (amant) qui fut tranchée par son propre mari et qui la force à l'embrasser deux fois par jour $^{38} »$. À ce moment, le mariage est devenu un sacrement, l'adultère et les mariages contre l'ordre naturel sont de plus en plus condamnés par les autorités religieuses et civiles. Cette double peine se retrouve sur les chapiteaux des cathédrales et les scènes de Jugement Dernier qui illustrent les péchés capitaux. L'iconographie de la luxure est directement inspirée des peines terrestres comme sur le portail occidental de SainteFoy de Conques ${ }^{39}$. On retrouve également une scène directement inspirée d'un texte populaire au XIII ${ }^{\mathrm{e}}$ siècle, Le Lai d'Aristote, sur le portail de la

\footnotetext{
${ }^{37}$ Michael Camille, L'Art de l'amour au Moyen Âge, op. cit., p. 16.

${ }^{38}$ Florence Colin-Goguel, L'Image de l'a mour charnel au Moyen Âge, op. cit., p. 30.

${ }^{39}$ Eliane Vergnolle, L'Art roman en France, Paris, Flammarion, 1994, p. 248-250. Pour l'histoire des péchés capitaux, consulter Carla Casagrande et Silvana Vecchio, Histoire des péchés capitaux au Moyen Âge, Paris, France, Flammarion, 2003.
} 
Calende à la cathédrale de Rouen. Cette scène est inspirée de la chevauchée d'Aristote : le philosophe est monté par sa jeune maîtresse indienne Phyllis comme un cheval. La scène sert de pendant à une image de Samson. Selon Denis Bruna, cette sculpture devait servir aux prédicateurs pour prévenir les fidèles contre la fourberie des femmes ${ }^{40}$. Ces images sont aussi présentes dans les manuscrits, comme sur le ms. fr. 2446, fol. 106r et le ms. fr. 5594, fol. 193v, conservés la Bibliothèque nationale de France. Sur ce premier folio, une nonne tire un moine par une corde attachée à son sexe. Dans certaines régions de France, cette condamnation était infligée publiquement aux adultères. Comme ces couples illégitimes nuisent à l'ordre divin et à l'ordre établi, on les représente dans une situation d'inversion des rôles, l'homme tenu en laisse $^{41}$. Sur la seconde miniature, c'est un mariage mal assorti qui est condamné : elle illustre un charivari au cours duquel un homme âgé, ayant épousé une femme jeune, est promené nu dans les rues, montant à l'envers sur un âne. L'âne symbolise la sottise, la folie et la violence sexuelle ${ }^{42}$.

\section{Un topos littéraire}

L'amour courtois développe une ambiguïté entre amour platonique et amour charnel. Dans la fin amor, on fustige les jeunes hommes qui s'habillent de façon trop efféminée pour séduire leur belle. Ils portent des vêtements moulants et ils sont surtout trop dénudés ${ }^{43}$. Mais, dans le même temps, la littérature développe des topoi qui vont contre l'ordre moral : le meilleur exemple en est sans doute le Roman de la Rose. Dans cet

\footnotetext{
${ }^{40}$ Denis Bruna, Saints et Diables au chapeau, op. cit., p. 82-86.

${ }^{41}$ Michael Camille, L'Art de l'amour au Moyen Âge, op. cit., p. 89-90.

${ }^{42}$ Florence Colin-Goguel, L'Image de l'amour charnel au Moyen Âge, op. cit., p. $166-168$.

${ }^{43}$ Ibid., p. 66-67.
} 
ouvrage, le jeune homme essaie de prendre d'assaut un jardin clos et un château fort symbolisant de la perte de virginité de la demoiselle. Tout commence avec le siège de la forteresse :

Vénus ne veut rien ajouter. Elle se retrousse, l'air irrité, tend l'arc [...] vise adroitement à une petite meurtrière qu'elle vit cachée dans la tour ; Nature l'avait placée, par une grande maîtrise, non pas sur le côté, mais sur le devant, entre deux petits piliers d'argent [...] c'est votre compagne et votre tendre amie qui vous attend et qui vous offre son amour, s'il vous plait de le recevoir ${ }^{44}$.

Toutefois, l'élément nouveau et important introduit par les poètes est que l'amour doit être gratuit et partagé. Cette conception a été développée par le christianisme avec les consentements mutuels des époux dans le mariage ${ }^{45}$.

Les fabliaux montrent, quant à eux, une forme de voyeurisme. L'érotique au Moyen Âge nous place souvent dans la position du voyeur de scènes licencieuses : «Et le vilain guettait à la porte et vit tout clairement : le cul de sa femme découvert et le prêtre dessus » (Le prêtre voyeur de Garin, XIII ${ }^{\mathrm{e}}$ siècle) ${ }^{46}$. Ces fabliaux présentent également des scènes beaucoup plus crues que celle de la fin amor, mais peut-être plus réalistes. Elles exposent souvent un triangle amoureux, comme chez les troubadours, mais les personnages y sont plus caricaturaux. Ainsi, dans ce fabliau, un prêtre et une femme mariée essaient de tromper le mari, en lui faisant croire que la scène érotique qu'il a surprise est un simple repas.

\footnotetext{
${ }^{44}$ Guillaume de Lorris et Jean de Meun, Le Roman de la Rose, éd. et trad. André Mary et Jean Dufournet Paris, Gallimard, 1984, p. 348 et 354.

${ }^{45}$ Arnaud de la Croix, L'Érotisme au Moyen Âge, le corps, le désir et l'amour, Paris, Tallandier, 1999, p. 43.

${ }^{46}$ Garin, Le prêtre voyeur, trad. Luciano Rossi, dans Fabliaux érotiques, Paris, Librairie Générale Française, 1992, p. 161.
} 
Au cours de cette scène, les deux complices tentent de prouver que cette méprise du mari serait due à un enchantement ${ }^{47}$.

La littérature courtoise assimile donc un certain nombre d'interdits religieux comme le viol ou l'adultère ; les fabliaux, quant à eux, les tournent en parodie afin d'amuser. Cette vision plus grivoise de l'amour est illustrée dans les arts, mais principalement dans la sphère privée et les milieux curiaux.

\section{La mise en place d'un art profane}

L'art profane puise discrètement aux sources de l'art religieux, en s'inspirant du premier couple pécheur que sont Adam et Ève. L'iconographie présente le plus souvent des couples non mariés et des couples adultères, à l'image de ceux présents dans les romans courtois. En effet, l'union dans le mariage est davantage spirituelle que charnelle, car les époux sont une incarnation terrestre du mariage spirituel du Christ Époux et de l'Église Épouse ${ }^{48}$.

Pour l'Église, l'adultère est essentiellement une faute féminine, qu'elle condamne avec une grande virulence, cette conception étant reprise dans les romans de chevalerie. Ainsi, ils décrivent essentiellement des femmes adultères qu'il s'agisse d'Yseult ou encore de la reine Guenièvre. L'adultère est un argument avancé contre le mariage indissoluble à la fin du XIII ${ }^{\mathrm{e}}$ siècle, car cette faute n'entraîne pas la rupture du sacrement ${ }^{49}$. Les enfants conçus par un couple adultère ou non-marié sont donc voués à la mort comme l'illustre un chapiteau du palais des Doges de Venise. Ce chapiteau fait d'ailleurs écho au couple d'Adam et Ève représenté sur la façade. Il montre parfaitement les étapes du

\footnotetext{
${ }^{47}$ Charles Baladier, Érôs au Moyen Âge, Amour, désir et délectation morose, Paris, Cerf, 1999, p. 141-146.

${ }^{48}$ Ibid., p. 116.

${ }^{49}$ Arnaud de la Croix, L'Érotisme au Moyen Âge, op. cit., p. 27 et 73-75.
} 
rapprochement amoureux : la vue, la conversation, l'attouchement, «l'échange de doux baisers» et «le degré ultime, le meilleur ${ }^{50}$ ». Cependant, la dernière scène voit la mort du fruit bâtard, car il a été conçu dans une union non consacrée par Dieu.

En Orient, ces scènes érotiques se développent essentiellement sur les objets profanes dans le contexte des cours impériales, sous couvert d'inspiration antique. Ainsi, à Byzance, sous la dynastie des Macédoniens, apparaissent des coffrets précieux en ivoire avec des putti nus faisant des gestes obscènes ${ }^{51}$. Un peu plus tard en Occident, une iconographie profane se déploie aussi sur des objets du quotidien comme des valves de miroir en os ${ }^{52}$; ils étaient souvent des cadeaux d'un homme à une femme pour l'inciter à lui céder. L'exemple le plus fameux est la valve de miroir conservée au musée du Louvre, présentant une partie d'échecs dont l'enjeu est une faveur sexuelle. Cet enjeu de la partie nous est indiqué par la position de la hampe par rapport au rideau, la main du jeune homme autour de celle-ci, la position de la cuisse de l'homme et surtout les plis de la robe de la femme qui conduit le regard vers la voie interdite $^{53}$.

La conception du plaisir représente aussi un point de basculement intéressant dans les théories puis dans les représentations artistiques. En effet, à la fin du Moyen Âge se développe la figure de la bonne et sainte épouse incarnée par le personnage de Marguerite de Hongrie ${ }^{54}$, qui se

\footnotetext{
${ }^{50}$ Michael Camille, L'Art de l'amour au Moyen Âge, op. cit., p. 13.

${ }^{51}$ Thomas F. Mathews, Le Monde byzantin, Paris, Flammarion, 2002, p. 84-85.

${ }^{52}$ Denis Bruna, Enseignes de plomb et autres menues chosettes du Moyen Âge, Paris, Le Léopard d'Or, 2006, p. 127 et p. 129-130. Cette évocation de l'amour apparaît sur des objets de plus modestes factures tels que des broches. L'iconographie de ces bijoux est plus simple, ainsi un faucon symbolise l'amant qui, à l'image de l'oiseau, fond sur sa proie.

${ }^{53}$ Michael Camille, L'Art de l'amour au Moyen Âge, op. cit., p. 124-125.

54 Edith Pásztor, «Margherita d'Ungheria, santa », dans Bibliotheca sanctorum, vol. VIII, Rome, Nuova Città Editrice, 1967, p. 796-800.
} 
soumet à ses devoirs conjugaux, non par plaisir, mais par nécessitée de donner des enfants. Cependant, progressivement, l'avancée de la médecine va faire évoluer cette conception, et la femme doit désormais éprouver du plaisir durant l'acte charnel, en raison de la théorie des humeurs. En effet, la médecine considère que le plaisir sexuel est indispensable à la bonne santé, comme un remède à la mélancolie, et chez les femmes, celui-ci serait nécessaire à la procréation ; sans lui elle ne pourrait pas secréter la substance nécessaire à la conception d'un être humain. Cette théorie est issue des médecins antiques Galien et Hipocrate ${ }^{55}$. Elle a engendré une production artistique jusqu'à la Renaissance. Selon 1'historien de l'art Daniel Arasse, c'est dans ce contexte qu'il faut analyser la Vénus d'Urbino de Titien, toile datée de 1538 et conservée à la Galerie des Offices de Florence. Il l'a qualifiée de «pin-up » : la fonction de ce tableau serait d'exciter l'homme et la femme. Il s'agit pour lui d'une femme nue sur un lit en train de se caresser le sexe. On recommande en effet d'accrocher des femmes nues dans la chambre des époux, car si la femme regardait de beaux corps au moment de la fécondation, les enfants seraient plus beaux et surtout elle donnerait naissance à des enfants mâles en bonne santé. La peinture érotique fleurit dans les milieux aristocratiques, mais elle est limitée au contexte matrimonial. $\mathrm{Au} \mathrm{XVI} \mathrm{e}^{\mathrm{e}}$ siècle, en raison de la théorie des humeurs, la masturbation féminine était recommandée, si le mari n'était pas très doué. Les prêtres conseillaient aussi cette pratique puisque la seule finalité de la sexualité dans le mariage est de concevoir des enfants. C'était un moyen de ne pas condamner le couple au péché de copulation sans progéniture ${ }^{56}$.

\footnotetext{
${ }^{55}$ Danielle Jacquart et Claude Thomasset, Sexualité et savoir médical au Moyen Âge, Paris, Presses universitaires de France, 1988, p. 88-89 et p. 180-184.

${ }^{56}$ Daniel Arasse, On n'y voit rien. Descriptions, Paris, Gallimard, 2000, « La femme dans le coffre », p. 123-173.
} 
Ce tableau du début de la Renaissance vénitienne et certaines images médiévales ont été délaissés longtemps par l'histoire de l'art, surtout au cours du pudibond $\mathrm{XIX}^{\mathrm{e}}$ siècle. Pour les historiens d'art, ces œuvres montraient un Moyen Âge obscène et vulgaire, car ils calquaient leurs propres réalités sociales et les décontextualisaient. Au contraire, il s'agit le plus souvent d'images à portée moralisatrice illustrant les débats d'idées, les interdits civiques, les dogmes religieux et la connaissance scientifique $^{57}$.

\section{Les pratiques}

Les pratiques sexuelles médiévales, pourrait-on croire, se laissent difficilement approcher derrière ces représentations. Mais les études menées depuis les années 1980 ont mis en lumière les liens étroits entre les pratiques sexuelles, le contrôle social et le contrôle politique à travers les interdits.

Ces pratiques ont en effet été règlementées par un autre corpus normatif : celui des lois civiles. Ces dernières et les procès qui en découlent nous permettent d'approcher de plus près la réalité de la sexualité, en particulier dans trois domaines particulièrement règlementés : le viol, la prostitution et l'homosexualité.

\section{Le viol}

Le viol est un interdit fondamental de la sexualité, même s'il n'a pas forcément la même signification qu'aujourd'hui ${ }^{58}$. Durant le haut Moyen Âge occidental, cet acte est prohibé car il est avant tout considéré comme un vol, une usurpation de la propriété du lignage sur la femme.

\footnotetext{
57 Ibid.

${ }^{58}$ La notion de consentement des deux parties étant relativement floue, de même que l'inégalité de traitement entre hommes et femmes est une constante.
} 
Dans les milieux nobiliaires, on considère davantage le rapt que le viol en tant que tel. Les cas de rapts créent « des couples qui ne se sont pas formés dans la paix » et pour cette raison, les ravisseurs doivent être poursuivis par le roi ${ }^{59}$. Mais il s'agit d'une pratique largement répandue comme en témoignent les récits des clercs des $\mathrm{XI}^{\mathrm{e}}$ et XII ${ }^{\mathrm{e}}$ siècles ${ }^{60}$.

Avec le changement de statut de la femme qui devient un sujet de droit, le viol change également de caractérisation. La relation sexuelle contrainte devient interdite d'un point de vue individuel, et non plus lignager. Cela ne veut bien sûr pas dire que le viol cesse d'être commis. Au contraire, à la fin du Moyen Âge, Élisabeth Crouzet-Pavan et Jacques Rossiaud ont constaté une situation d'insécurité pour les femmes, surtout les plus fragiles, en Italie du Nord et dans le Sud de la France. Il s'agit d'exactions de bandes de jeunes hommes laissés pour compte du mariage qui commettent des agressions nocturnes sur les femmes pauvres, sans dot, ou veuves. Le contexte socio-économique et démographique de l'époque doit être pris en compte, en particulier un marché matrimonial auquel il devient parfois difficile d'accéder. Toujours est-il que selon Élisabeth Crouzet-Pavan, il existe « une infraculture du viol au sein de laquelle l'immédiate possession du corps féminin, par la force, fait partie du registre des conduites sexuelles ${ }^{61} »$. Il s'agit bien d'un interdit, réprimé et poursuivi, mais qui pourtant fait partie d'une certaine norme de la

\footnotetext{
${ }^{59}$ Georges Duby, Le Chevalier, la femme et le prêtre, op. cit., p. 43-44.

${ }^{60}$ Le rapt peut être simulé pour libérer un mari de sa femme, pour priver une sœur de son héritage, pour s'épargner les frais d'une cérémonie, ou encore pour forcer le consentement des parents.

${ }^{61}$ Élisabeth Crouzet-Pavan, «Femmes et jeunes : sur les liaisons dangereuses dans l'Italie de la fin du Moyen Âge ", dans Mariage et sexualité au Moyen Âge, dir. Michel Rouche, Paris, PUPS, 2000, p. 287-300, cit. p. 295. Sur le phénomène des bandes de jeunes, on renvoie également à Ilaria Taddei, Faciulli e giovani. Crescere a Firenze nel Rinascimento, Florence, Olschki, 2001 ; pour les rapports de ceux-ci à la prostitution, voir Jacques Rossiaud, «Prostitution, jeunesse et société dans les villes du Sud-Est au Xv ${ }^{\mathrm{e}}$ siècle », Annales. Économies, Sociétés, Civilisations, vol. 31, $\mathrm{n}^{\circ} 2$, 1976, p. 289-325.
} 
virilité au Moyen Âge, qui valorise les exploits sexuels souvent violents $^{62}$. La répression du viol répond donc toujours à des impératifs sociaux, mais sa pratique même sanctionne des hiérarchies, entre homme et femme bien sûr, mais aussi entre différentes catégories de la population plus ou moins fragiles.

\section{La prostitution}

Certains auteurs constatent le changement de considération du viol dans sa pénalisation quand il concerne des prostituées qui deviennent à la fin du Moyen Âge des sujets de droit, susceptibles de porter plainte pour viol comme n'importe quelle autre femme honnête. La vie d'une femme vénale est bien entendu réprouvée par les autorités religieuses comme civiles. Cependant, la prostitution est un mal nécessaire ${ }^{63}$. À partir de 1100, les meretrices publicae, loin d'être marginales, sont omniprésentes dans les récits, les représentations, et dans la réalité quotidienne des villes $^{64}$. Elles sont identifiées par des marques vestimentaires pour éviter toute confusion. Ainsi à Toulouse, elles protestent contre la trop grande visibilité des signes qui leur échoient ${ }^{65}$. Dans ce cadre, les politiques urbaines ségrègent puis institutionnalisent les prostituées, créant à partir de 1300 des prostibulae publicae : il s'agit finalement de «contrôler et

\footnotetext{
${ }^{62}$ Claude Thomasset, «Le médiéval, la force et le sang », dans Histoire de la virilité, dir. Georges Vigarello, Paris, Éditions du Seuil, 2011, vol. 1, p. 141-181, p. 151-152 et p. 156. Durant certaines fêtes, des simulacres de rapt et de viol sont commis par des hommes déguisés en ours, qui devient l'image archétypale de la virilité.

${ }^{63}$ Suivant saint Jérôme et saint Augustin, certains théologiens considèrent qu'il est moins répréhensible d'aller voir les prostituées que d'être amant trop fougueux de sa femme, car, ce faisant, le mari souille une femme a priori honnête : la prostitution permet de ne pas dévergonder le mariage devenu un sacrement : Jacques Rossiaud, Amours vénales, op. cit., p. 37-38. On se réfèrera à la bibliographie sur la prostitution dans l'Occident médiéval de cet ouvrage.

${ }^{64}$ Ibid., p. 36-37.

${ }^{65}$ Charles VI décide alors d'assouplir ces marques distinctives : Ordonnances des Rois de France de la troisième race, t. VII, Paris, Imprimerie nationale, 1848, p. 327 ; voir aussi, ibid., t. XIII, p. 75.
} 
profiter du phénomène sans le réprimer ${ }^{66}{ }$. De même, la prostitution est très tôt contrôlée en Islam avec des maisons de tolérance et une taxation officielle mise en place notamment dans l'Égypte fatimide ${ }^{67}$.

Où est l'interdit alors, quand la fin du Moyen Âge et le début de l'époque moderne voient apparaître des figures de courtisanes splendides et bien en vue? C'est l'excès que l'on réprime plus que les faits ponctuels. Sans doute l'interdit est-il dans l'excès. Si les hommes mariés ne sont pas interdits de maisons closes, ceux-ci ne sont cependant pas censés y aller trop souvent, au risque de se voir qualifiés de « concubinaires de bordel » et parfois poursuivis pour adultère. Les juifs, les lépreux, les adolescents et les «putiers », ou hommes de mauvaise vie, en sont aussi exclus ${ }^{68}$. Par ailleurs, le vent tourne au début du $\mathrm{XVI}^{\mathrm{e}}$ siècle : des interdictions municipales touchent la prostitution, par peur du mal de Naples et sous l'influence morale de la Réforme ${ }^{69}$. Enfin, si les femmes prostituées sont considérées comme accoutumant les hommes à l'amour licite, les garçons qui se vendent « a uso di donna », comme c'est le cas dans certaines cités italiennes, et surtout leurs clients, sont poursuivis et sévèrement condamnés.

La prostitution répond donc finalement à un impératif social et politique. Elle est autorisée en tant qu'elle permet l'équilibre de la société ; elle est réprouvée quand elle le perturbe. Quand elle se mêle à l'homosexualité, qui est perçue à la fin du Moyen Âge comme un retournement complet de l'ordre naturel et social, elle est fondamentalement interdite et réprimée.

\footnotetext{
${ }^{66}$ Ibid., p. 45.

${ }^{67}$ Abdelwahab Bouhdiba, La Sexualité en islam, op. cit., p. 228-231.

${ }^{68}$ Jacques Rossiaud, Amours vénales, op. cit., p. 181.

${ }^{69}$ Ibid., p. 293-294. Cela n'empêche pas l'amour vénal de perdurer, clandestinement ou sous d'autres formes.
} 


\section{L'homosexualité}

Les relations homosexuelles semblent également assez répandues en Islam, particulièrement dans les élites abbassides, malgré la condamnation religieuse. Du côté de l'Occident, l'homosexualité masculine n'est pas la principale préoccupation des clercs au haut Moyen Âge. L'amour entre hommes ne devient un réel problème qu'à partir des XIII ${ }^{\mathrm{e}}-\mathrm{XIV}^{\mathrm{e}}$ siècles $^{70}$. La répression est organisée par les autorités publiques. Un fuero en Castille punit la sodomie de la castration et du bûcher; les coutumiers français et les lois communales italiennes oscillent entre le bannissement et le bûcher; $a u X V^{e}$ siècle en Italie, des institutions ad hoc sont créées ${ }^{71}$. La pratique semble largement répandue : à Florence, entre 1432 et 1502, 15 à 16000 individus sont impliqués dans des affaires de sodomies et entre 2400 et 3000 sont condamnées ${ }^{72}$. À Venise, la pratique est bien attestée dans les archives. Mais on peut se demander s'il ne s'agit pas d'un effet de source. Élisabeth Crouzet-Pavan a ainsi supposé que les accusations de sodomie touchaient particulièrement les artisans et les patriciens parce qu'il s'agissait des catégories de la population les plus surveillées par les autorités et les plus

\footnotetext{
${ }^{70}$ La question de 1'homosexualité masculine au Moyen Âge, et de l'existence ou non d'une culture homosexuelle ou hétérosexuelle, a fait l'objet de débats vifs dans l'historiographie du genre. Citons en particulier Guido Ruggiero, pour qui il existe une sous-culture homosexuelle (Guido Ruggiero, The Boundaries of Eros : sex crime and sexuality in Renaissance Florence, New York/Oxford, Oxford University Press, 1985) ; Michael Rocke, pour qui l'homosexualité est une étape normale dans la vie d'un jeune Florentin (Michael Rocke, Forbidden friendship. Homosexuality and male culture in Renaissance Florence, Oxford, Oxford University Press, 1998) ; et enfin, Louis-Georges Tin dont l'ouvrage, très critiqué, cherche à démontrer l'invention d'une culture hétérosexuelle à travers la culture courtoise, alors que l'ethos chevaleresque aurait davantage tendu vers l'amour viril entre hommes (Luis-Georges Tin, L'Invention de la culture hétérosexuelle, Paris, Autrement, 2008). Pour une excellente recension critique de cet ouvrage, on se réfèrera au compte-rendu publié par Didier Lett, Clio, vol. 31, 2010, p. 287-290.

${ }^{71}$ L'Onestà puis l'Officio della notte à Florence en 1432 ; à Venise, le Collegium sodomitarum en 1418. La réalité se laisse apercevoir par les procès, très nombreux : Jacques Rossiaud, Amours vénales, op. cit., p. 286-287.

${ }^{72}$ Ibid., p. 136.
} 
à risque pour l'ordre social et politique ${ }^{73}$. Puisque l'homosexualité est pensée comme perturbant l'ordre social, cette accusation est également retournée contre des groupes qui sont soupçonnés d'œuvrer contre l'équilibre politique. À l'inverse, l'homosexualité féminine attire bien moins l'attention et est moins perçue comme problématique : des rapports entre deux femmes n'impliquent pas de gaspillage de sperme et celles-ci sont censées revenir naturellement à l'amour des hommes ${ }^{74}$. De telles affaires sont donc très peu présentes dans les archives judiciaires. Cette absence de considération, tout comme la méfiance qui entourait l'homosexualité masculine, révèle donc également une certaine perception des rapports entre hommes et femmes. La sexualité et ses interdits ne sont jamais neutres et participent d'une vision d'ensemble de la société.

\section{Des pratiques déviantes?}

Au-delà de ces trois domaines pris en charge par les autorités publiques, que sait-on des pratiques privées? Certaines pratiques déviantes se laissent apercevoir, mais avec parcimonie.

Nous pouvons ainsi considérer que les interdits répertoriés avec précision dans certaines sources, comme les pénitentiels, ou les déviances présentées dans la littérature ou les arts, ne sortaient pas seulement de l'esprit pervers des moines, des poètes ou des artistes. Elles pouvaient également avoir une forme de réalité : telle la répression farouche de la «bestialité » ou de la zoophilie qui occupe une place importante dans les pénitentiels du haut Moyen Âge, et qui devait sans doute représenter une

\footnotetext{
${ }^{73}$ Élisabeth Crouzet-Pavan, «Police des mœurs, société et politique à Venise à la fin du Moyen Âge », Revue historique, vol. 536, 1980, p. 241-288.

${ }^{74}$ Jacques Rossiaud, Sexualités au Moyen Âge, op. cit., p. 68-69.
} 
réalité tangible dans un monde essentiellement rural $^{75}$. Ces mêmes sources nous laissent voir toute la variété des possibilités de masturbation, pratique qui est pourtant très fermement condamnée par l'Église jusqu'au bas Moyen Âge, pour les hommes en tout cas ${ }^{76}$. La pédophilie quant à elle, comme on a pu le voir, est bien souvent confondue avec l'homosexualité masculine à la fin du Moyen Âge, avec ces garçons qui pratiquent l'amour vénal, notamment dans les cités italiennes. D'autres textes montrent cependant que la pédophilie pouvait être une pratique courante dans d'autres cadres, bien que condamnée. Ainsi Jean de Phobéros dans son Typikon mentionne avec force détails les tentations qu'un moine de sa connaissance a eu à subir, en tant que moine, à la vue d'un jeune enfant de 10 ans ; il rapporte sa lutte héroïque contre le démon, et justifie ainsi le fait d'exclure la présence d'enfants dans l'enceinte du monastère ; il rapporte également que cette tentation est courante, y compris chez des moines exempts de tout soupçon qui peuvent souvent céder à ce péché ${ }^{77}$.

Même dans les normes les plus ancrées, on voit que la pratique s'écarte souvent : l'adultère est condamné théoriquement, mais la réalité sociale est bien plus permissive. Il est même largement admis que des couples puissent vivre comme mari et femme sans être passés par les

\footnotetext{
${ }^{75}$ Ainsi une quittance du 26 août 1457, atteste du paiement à Jean Fouillet, maître et exécuteur de la haute justice, de 30 sous tournois pour avoir brulé Guillaume Nourrissier, natif de Bretagne et qui avait eu «habitattion charnelle » avec une jument. Il est également payé 10 sous tournois pour l'exécution de la jument. La quittance est conservée à la bibliothèque municipale de Rouen (Leber 5704).

${ }^{76}$ Jean-Louis Flandrin, Un Temps pour embrasser, op. cit. La masturbation peut conduire au dépeuplement et favoriserait l'homosexualité ; Jean Gerson en fait un «péché pire que manger chair au Grand Vendredi »: Jacques Rossiaud, Amours vénales, op. cit.

77 Jean de Phobéros, Typikon, dans Noctes Petropolitanae, éd. Anastasis Papadopoulos-Kerameus, Leipzig, Zentralantiquariat der Deutschen Demokratischen Republik, 1976, cité dans Économie et société à Byzance, $\mathrm{VIII}^{\mathrm{e}}-\mathrm{XII}{ }^{\mathrm{e}}$ siècle : textes et documents, dir. Sophie Métivier et Paule Pagès, Paris, Publications de la Sorbonne, 2007, p. 209-211.
} 
sacrements du mariage, et ce même tard au Moyen Âge: Montaillou comptait environ $10 \%$ de couples non sanctifiés ${ }^{78}$. La fin du Moyen Âge voit également la disparition de certains châtiments infamants contre les époux adultères, preuve sans doute d'une plus grande tolérance au fait, surtout quand il s'agit des hommes... En Islam, les ouvrages littéraires ou juridiques nous signalent des pratiques très libres en matière de concubinat $^{79}$, avec en particulier les jawari, de véritable anti-épouses concubines, souvent esclaves, qui acquièrent un statut social reconnu par le fait d'être mère des enfants du maître. Ces femmes très savantes et cultivées, d'origines et d'ethnies diverses, ont largement contribué à l'acculturation de la société arabo-islamique à travers leur présence dans les harems des califes et hauts dignitaires omeyyades ou abbassides.

$\mathrm{Au}$ terme de cette trop rapide introduction, il nous faut finalement rejeter l'image d'un Moyen Âge fixe, dont la sexualité ne serait faite que d'interdictions et dont le but ne serait que reproductif. Non seulement les pratiques sont diverses, mais même les textes normatifs s'infléchissent et doivent se comprendre dans leur évolution: les normes du Coran, des Pères de l'Église ou de la Kabbale sont interprétées diversement selon les époques et les lieux, par des juristes ou des théologiens plus ou moins rigoristes. Et ceux-ci sont d'ailleurs bien conscients des limites de leurs prescriptions : Pierre le Chantre finit par conclure qu'il n'y ni culpabilité à avoir, ni péché véniel tant que les couples se tiennent loin des excès et du plaisir immodéré de la luxure ${ }^{80}$.

\footnotetext{
${ }^{78}$ Emmanuel Le Roy Ladurie, Montaillou, village occitan de 1294 à 1324, Paris, Gallimard, 1975, p. 242.

${ }^{79}$ Abdelwahab Bouhdiba, La Sexualité en islam, op. cit., p. 130.

${ }^{80}$ Pierre le Chantre, À I Co. VII, 5, Paris, Mazarine, ms. 176, fol. $108 \mathrm{r}^{\circ} \mathrm{b}$, cité dans John Baldwin, Les Langages de l'amour dans la France de Philippe Auguste, Paris, Fayard, 1997, p. 196.
} 